Full-field drift Hamiltonian particle orbits in 3D geometry

This article has been downloaded from IOPscience. Please scroll down to see the full text article.

2011 Plasma Phys. Control. Fusion 53024001

(http://iopscience.iop.org/0741-3335/53/2/024001)

View the table of contents for this issue, or go to the journal homepage for more

Download details:

IP Address: 128.178.125.164

The article was downloaded on 04/02/2011 at 14:54

Please note that terms and conditions apply. 


\title{
Full-field drift Hamiltonian particle orbits in 3D geometry
}

\author{
W A Cooper ${ }^{1}$, J P Graves ${ }^{1}$, S Brunner ${ }^{1}$ and M Yu Isaev ${ }^{2}$ \\ ${ }^{1}$ Ecole Polytechnique Fédérale de Lausanne (EPFL), Centre de Recherches en Physique des \\ Plasmas, Association Euratom-Confédération Suisse, CH1015 Lausanne, Switzerland \\ ${ }^{2}$ Nuclear Fusion Institute, RRC 'Kurchatov Institute', 123182 Moscow, Russia \\ E-mail: wilfred.cooper@epfl.ch
}

Received 11 June 2010, in final form 9 July 2010

Published 20 January 2011

Online at stacks.iop.org/PPCF/53/024001

\begin{abstract}
A Hamiltonian/Lagrangian theory to describe guiding centre orbit drift motion which is canonical in the Boozer coordinate frame has been extended to include full electromagnetic perturbed fields in anisotropic pressure 3D equilibria with nested magnetic flux surfaces. A redefinition of the guiding centre velocity to eliminate the motion due to finite equilibrium radial magnetic fields and the choice of a gauge condition that sets the radial component of the electromagnetic vector potential to zero are invoked to guarantee that the Boozer angular coordinates retain the canonical structure. The canonical momenta are identified and the guiding centre particle radial drift motion and parallel gyroradius evolution are derived. The particle coordinate position is linearly modified by wave-particle interactions. All the nonlinear wave-wave interactions appear explicitly only in the evolution of the parallel gyroradius. The radial variation of the electrostatic potential is related to the binormal component of the displacement vector for MHD-type perturbations. The electromagnetic vector potential projections can then be determined from the electrostatic potential and the radial component of the MHD displacement vector.
\end{abstract}

\section{Introduction}

Numerical schemes for modelling guiding centre particle motion are facilitated when the coordinate frame invoked is canonical because the tools of nonlinear Hamiltonian dynamics become applicable [1,2]. Auxiliary heating schemes for magnetically confined plasmas can cause the energetic particle distribution to deviate significantly from a Maxwellian which results in a pressure anisotropy in the equilibrium state [3-7]. This can modify the guiding centre drift trajectories [8-10]. The application of the Lagrangian formalism constitutes an invaluable tool to identify canonical coordinates $[11,12]$. In general geometry, it is practical to redefine the 
guiding centre velocity to eliminate the motion associated with the finite radial component of the equilibrium magnetic field in the covariant representation $[12,13]$. In axisymmetric geometry, this elimination is in fact superfluous $[14,15]$. The derivation of the drift motion with full electromagnetic fields has been presented in coordinates which are impractical for application purposes [9, 16]. In the Boozer coordinate frame, it has been outlined by White [12] and partially extended for axisymmetric geometry [13]. A derivation in nonstraight field line coordinates has also been reported [17]. In this work, we extend the Hamiltonian drift orbit theory to full electromagnetic fields in three-dimensional (3D) anisotropic pressure equilibrium geometry. Furthermore, we investigate the relationship of the perturbed fields required for orbit evolution with those computed by magnetohydrodynamic (MHD) stability programmes.

\section{The anisotropic pressure MHD equilibrium state}

The MHD equilibrium state with parallel $p_{\|}(s, B)$ and perpendicular $p_{\perp}(s, B)$ pressure anisotropy, where $0 \leqslant s \leqslant 1$ is the radial variable and $B$ is the magnetic field strength, is given by [18-20]

$$
\left.\frac{\partial p_{\|}}{\partial s}\right|_{B} \nabla s=\boldsymbol{K} \times \boldsymbol{B},
$$

where the current density $\boldsymbol{K}$ is given by

$$
\mu_{0} \boldsymbol{K} \equiv \boldsymbol{\nabla} \times \boldsymbol{H},
$$

in which the magnetic intensity $\boldsymbol{H}=\sigma \boldsymbol{B}$ and the effective permeability of the plasma is $\mu_{0} / \sigma$. We see from equation (1) that $\boldsymbol{K} \cdot \nabla s=0$, so that the current density lines lie on the flux surfaces. The firehose stability criterion is [18]

$$
\sigma \equiv 1-\left.\frac{\mu_{0}}{B} \frac{\partial p_{\|}}{\partial B}\right|_{s}=1-\frac{\mu_{0}\left(p_{\|}-p_{\perp}\right)}{B^{2}}>0,
$$

and the mirror stability criterion is [18]

$$
\left.\tau \equiv \frac{\partial(\sigma B)}{\partial B}\right|_{s}=1+\left.\frac{\mu_{0}}{B} \frac{\partial p_{\perp}}{\partial B}\right|_{s}>0
$$

\section{The magnetic field and the vector potential in the Boozer frame}

In the Boozer magnetic coordinates [1], the magnetic field lines are straight which in the contravariant representation is expressed as

$$
\boldsymbol{B}=\nabla \varphi \times \nabla \psi(s)+\nabla \chi(s) \times \nabla \vartheta,=\nabla \times[\chi(s) \nabla \vartheta-\psi(s) \nabla \varphi],
$$

where $\vartheta$ and $\varphi$ are the poloidal and toroidal angles, while $\psi(s)$ and $\chi(s)$ are the poloidal and toroidal magnetic flux functions, respectively. The equilibrium magnetic field vector potential corresponds to $\boldsymbol{A}_{\mathrm{e}}=\chi(s) \nabla \vartheta-\psi(s) \boldsymbol{\nabla} \varphi$. In the covariant representation

$$
\sigma \boldsymbol{B}=\mu_{0} J(s) \nabla \vartheta-\mu_{0} I(s) \nabla \varphi+\sigma B_{s} \nabla s .
$$

The toroidal and poloidal current fluxes are $J(s)$ and $I(s)$, respectively. The total vector potential (equilibrium + perturbed) is expressed as

$$
\begin{aligned}
\boldsymbol{A}=\boldsymbol{A}_{\mathrm{e}}+\delta \boldsymbol{A} & =\left[\chi(s)+\delta A_{\vartheta}(s, \vartheta, \varphi, t)\right] \nabla \vartheta-\left[\psi(s)-\delta A_{\varphi}(s, \vartheta, \varphi, t)\right] \nabla \varphi \\
& \left.+\delta A_{s}(s, \vartheta, \varphi, t)\right] \nabla s .
\end{aligned}
$$


We shall see that it is very convenient to write the following vector potential components:

$\Upsilon(s, \vartheta, \varphi, t)=\frac{\boldsymbol{B} \cdot \delta \boldsymbol{A}}{\sigma B^{2}}=\frac{\psi^{\prime}(s) \delta A_{\vartheta}(s, \vartheta, \varphi, t)+\chi^{\prime}(s) \delta A_{\varphi}(s, \vartheta, \varphi, t)}{\sigma \sqrt{g} B^{2}}$,

$\mathcal{W}(s, \vartheta, \varphi, t)=-\frac{\sqrt{g} \sigma \boldsymbol{B} \times \nabla s \cdot \delta \boldsymbol{A}}{\mu_{0} I(s)}=\delta A_{\vartheta}(s, \vartheta, \varphi, t)+\frac{J(s)}{I(s)} \delta A_{\varphi}(s, \vartheta, \varphi, t)$.

From the expressions of the magnetic field in the covariant and contravariant representation, it is trivial to derive the relation $\sigma \sqrt{g} B^{2}=\mu_{0}\left[\psi^{\prime}(s) J(s)-\chi^{\prime}(s) I(s)\right]$, where $\sqrt{g}$ is the Jacobian of the transformation from Cartesian coordinates to the anisotropic Boozer frame.

\section{The Lagrangian and canonical coordinates}

The momentum vector in the drift approximation is

$$
\boldsymbol{P}=P_{\|} \frac{\boldsymbol{B}}{\boldsymbol{B}}+e \boldsymbol{A},
$$

where $e$ is the particle charge. The parallel gyroradius is given by

$$
\rho_{\|}=\frac{P_{\|}}{e \sigma B},
$$

and we find it convenient to define the following effective parallel gyroradii:

$$
\begin{aligned}
& \rho_{c} \equiv \rho_{\|}+\Upsilon(s, \vartheta, \varphi, t), \\
& \rho_{W} \equiv \rho_{c}-\frac{\psi^{\prime}(s)}{\sigma \sqrt{g} B^{2}} \mathcal{W}(s, \vartheta, \varphi, t)=\rho_{\|}-\frac{\delta A_{\varphi}}{\mu_{0} I(s)} .
\end{aligned}
$$

The Lagrangian for the guiding centre motion then is

$$
\begin{aligned}
\mathcal{L} \mathrm{d} t & =\boldsymbol{P} \cdot \mathrm{d} \boldsymbol{x}-\mathcal{H} \mathrm{d} t, \\
& =e\left(\rho_{\|} \sigma \boldsymbol{B}+\boldsymbol{A}\right) \cdot \mathrm{d} \boldsymbol{x}-\mathcal{H} \mathrm{d} t,
\end{aligned}
$$

where we denote the Lagrangian by $\mathcal{L}$ and the Hamiltonian by $\mathcal{H}$ while the differential length vector is

$$
\mathrm{d} \boldsymbol{x}=\sqrt{g} \nabla s \times \nabla \vartheta \mathrm{d} \varphi+\sqrt{g} \nabla \varphi \times \nabla s \mathrm{~d} \vartheta+\sqrt{g} \nabla \vartheta \times \nabla \varphi \mathrm{d} s .
$$

Expansion of the Lagrangian yields

$$
\begin{gathered}
\frac{\mathcal{L} \mathrm{d} t}{e}=\left[\rho_{\|} \sigma B_{s}+\delta A_{s}(s, \vartheta, \varphi, t)\right] \mathrm{d} s+\left[\chi(s)+\rho_{\|} \mu_{0} J(s)+\delta A_{\vartheta}(s, \vartheta, \varphi, t)\right] \mathrm{d} \vartheta \\
-\left[\psi(s)+\rho_{\|} \mu_{0} I(s)-\delta A_{\varphi}(s, \vartheta, \varphi, t)\right] \mathrm{d} \varphi-\frac{\mathcal{H} \mathrm{d} t}{e} .
\end{gathered}
$$

This does not represent a canonical description of the drift orbit motion for finite $\rho_{\|} \sigma B_{s}+\delta A_{s}$. A means to eliminate this term must be determined. It can be accomplished by redefining the guiding centre velocity $[12,13]$ so that $\mathrm{d} \boldsymbol{x} \Rightarrow \mathrm{d} \boldsymbol{x}+\boldsymbol{w} \mathrm{d} t$ and given that the particle velocity along the magnetic field lines is much larger than the drift velocity it is appropriate to choose $\boldsymbol{w}=\lambda \boldsymbol{B}$. Then the Lagrangian reduces to

$$
\mathcal{L} \mathrm{d} t=\boldsymbol{P} \cdot \mathrm{d} \boldsymbol{x}+\boldsymbol{P} \cdot \boldsymbol{w} \mathrm{d} t-\mathcal{H} \mathrm{d} t,
$$

which becomes

$$
\begin{aligned}
\frac{\mathcal{L} \mathrm{d} t}{e}=\left[\rho_{\|} \sigma B_{s}\right. & \left.+\delta A_{s}(s, \vartheta, \varphi, t)\right] \mathrm{d} s+\left[\chi(s)+\rho_{\|} \mu_{0} J(s)+\delta A_{\vartheta}(s, \vartheta, \varphi, t)\right] \mathrm{d} \vartheta \\
- & {\left[\psi(s)+\rho_{\|} \mu_{0} I(s)-\delta A_{\varphi}(s, \vartheta, \varphi, t)\right] \mathrm{d} \varphi-\frac{\mathcal{H} \mathrm{d} t}{e} } \\
+ & \lambda\left[\rho_{\|} \sigma B^{2}+\delta \boldsymbol{A} \cdot \boldsymbol{B}\right] \mathrm{d} t .
\end{aligned}
$$


A judicious choice for $\lambda$ can ensure that the Lagrangian retains only angular coordinate variations and thus achieve canonicity. One option, as proposed by White [12], is to select

$$
\left[\rho_{\|} \sigma B_{s}+\delta A_{s}(s, \vartheta, \varphi, t)\right] \mathrm{d} s=\lambda\left[\rho_{\|} \sigma B^{2}+\delta \boldsymbol{A} \cdot \boldsymbol{B}\right] \mathrm{d} t .
$$

But we find this unsatisfactory because following the standard derivation we see that when we try to evaluate the perturbed radial magnetic field we are naturally led towards the imposition of a gauge condition on $\delta \boldsymbol{A}$ that annihilates $\mathcal{W}(s, \vartheta, \varphi, t)$ which corresponds exactly to the model where only $\delta A_{\|}$contributions are retained. A more favourable option is to choose

$$
\lambda=-\frac{\rho_{\| \mid} \sigma B_{s}}{\rho_{\|} \sigma B^{2}+\delta \boldsymbol{A} \cdot \boldsymbol{B}} \frac{\mathrm{d} s}{\mathrm{~d} t} .
$$

Then the Lagrangian becomes

$$
\begin{aligned}
\frac{\mathcal{L} \mathrm{d} t}{e}=[\chi(s) & \left.+\rho_{\|} \mu_{0} J(s)+\delta A_{\vartheta}(s, \vartheta, \varphi, t)\right] \mathrm{d} \vartheta-\frac{\mathcal{H} \mathrm{d} t}{e} \\
& -\left[\psi(s)+\rho_{\|} \mu_{0} I(s)-\delta A_{\varphi}(s, \vartheta, \varphi, t)\right] \mathrm{d} \varphi+\delta A_{s}(s, \vartheta, \varphi, t) \mathrm{d} s .
\end{aligned}
$$

This form for the Lagrangian satisfies the conditions for a canonical representation of the drift orbits if and only if we invoke the gauge condition

$$
\delta A_{s}(s, \vartheta, \varphi, t)=0,
$$

which we are free to exercise.

We can identify the canonical momenta from the expression derived from the Lagrangian as

$$
\begin{aligned}
& P_{\varphi}=-\psi(s)-\rho_{\|} \mu_{0} I(s)+\delta A_{\varphi}(s, \vartheta, \varphi, t), \\
& P_{\vartheta}=\chi(s)+\rho_{\| \mid} \mu_{0} J(s)+\delta A_{\vartheta}(s, \vartheta, \varphi, t) .
\end{aligned}
$$

The corresponding canonical angular variables are $\vartheta$ and $\varphi$, the Boozer angles. It is convenient to define $\mathcal{T} \equiv-\delta A_{\varphi} /\left[\mu_{0} I(s)\right]$ and use $\rho_{W}=\rho_{\|}+\mathcal{T}$ to obtain

$$
\begin{aligned}
& P_{\varphi}=-\psi(s)-\rho_{W} \mu_{0} I(s), \\
& P_{\vartheta}=\chi(s)+\rho_{W} \mu_{0} J(s)+\mathcal{W}(s, \vartheta, \varphi, t) .
\end{aligned}
$$

This reduction is appropriate for stellarators where $J$ can vanish and tokamak systems with inductive current drive and current reversal where $J$ may vanish locally, but is not justifiable for reversed field pinches because $\mathcal{T}$ becomes undefined at the field reversal point. Inversion of the canonical momenta suggests that

$$
\begin{aligned}
& s=s\left(P_{\vartheta}, P_{\varphi}, \vartheta, \varphi\right), \\
& \rho_{W}=\rho_{W}\left(P_{\vartheta}, P_{\varphi}, \vartheta, \varphi\right) .
\end{aligned}
$$

\section{The Hamiltonian formalism}

The Hamiltonian for the guiding centre drift motion is

$$
\frac{\mathcal{H}}{e}=\frac{e \rho_{\|}^{2} \sigma^{2} B^{2}}{2 m_{0}}+\frac{\mu B}{e}+\Phi_{\mathrm{E}}(s, \vartheta, \varphi, t)=\mathcal{H}_{e},
$$

where $m_{0}$ is the particle mass, $\mu$ its magnetic moment, $\Phi_{\mathrm{E}}$ is the electrostatic potential and the corresponding equations of motion are

$$
\begin{array}{ll}
\dot{P}_{\vartheta}=-\left.\frac{\partial \mathcal{H}_{e}}{\partial \vartheta}\right|_{P_{\vartheta}, P_{\varphi}, \varphi, t}, & \dot{\vartheta}=\left.\frac{\partial \mathcal{H}_{e}}{\partial P_{\vartheta}}\right|_{P_{\varphi}, \vartheta, \varphi, t}, \\
\dot{P}_{\varphi}=-\left.\frac{\partial \mathcal{H}_{e}}{\partial \varphi}\right|_{P_{\vartheta}, P_{\varphi}, \vartheta, t}, & \dot{\varphi}=\left.\frac{\partial \mathcal{H}_{e}}{\partial P_{\varphi}}\right|_{P_{\vartheta}, \vartheta, \varphi, t} .
\end{array}
$$


Here $\dot{\mathcal{A}} \equiv \mathrm{d} \mathcal{A} / \mathrm{d} t$. Applying the relations derived in the appendix, the equations of motion for the canonical momenta reduce to

$$
\begin{aligned}
& \dot{P}_{\vartheta}=-\left.\left(\frac{\mu}{e}+\sigma \tau \frac{e B}{m_{0}} \rho_{\|}^{2}\right) \frac{\partial B}{\partial \vartheta}\right|_{s, \varphi}-\left.\frac{\partial \Phi_{\mathrm{E}}}{\partial \vartheta}\right|_{s, \varphi, t}+\left.\frac{e \sigma^{2} B^{2}}{m_{0}} \rho_{\|} \frac{\partial \mathcal{T}}{\partial \vartheta}\right|_{s, \varphi, t}+\left.\frac{\partial \mathcal{W}}{\partial \vartheta}\right|_{s, \varphi, t} \dot{\vartheta} \\
& \dot{P}_{\varphi}=-\left.\left(\frac{\mu}{e}+\sigma \tau \frac{e B}{m_{0}} \rho_{\|}^{2}\right) \frac{\partial B}{\partial \varphi}\right|_{s, \vartheta}-\left.\frac{\partial \Phi_{\mathrm{E}}}{\partial \varphi}\right|_{s, \vartheta, t}+\left.\frac{e \sigma^{2} B^{2}}{m_{0}} \rho_{\|} \frac{\partial \mathcal{T}}{\partial \varphi}\right|_{s, \vartheta, t}+\left.\frac{\partial \mathcal{W}}{\partial \varphi}\right|_{s, \vartheta, t} \dot{\vartheta}
\end{aligned}
$$

where the subscripts indicate which variables are kept fixed during the evaluation of the partial derivatives. This representation for the evolution of the canonical momenta has the utility that it easily reduces to the equations previously derived using the finite $\delta A_{\|}$only model by dropping the terms with $\mathcal{W}$ and replacing $\mathcal{T}$ with $\Upsilon$. It is, however, much more convenient and of greater direct relevance to follow the evolution of the radial particle position and its parallel gyroradius rather than the momenta. With the application of the chain rule, we note that

$$
\begin{aligned}
& \dot{s}=\left.\frac{\partial s}{\partial P_{\vartheta}}\right|_{P_{\varphi}, \vartheta, \varphi} \dot{P_{\vartheta}}+\left.\frac{\partial s}{\partial P_{\varphi}}\right|_{P_{\vartheta}, \vartheta, \varphi} \dot{P}_{\varphi}+\left.\frac{\partial s}{\partial \vartheta}\right|_{P_{\vartheta}, P_{\varphi}, \varphi} \dot{\vartheta}+\left.\frac{\partial s}{\partial \varphi}\right|_{P_{\vartheta}, P_{\varphi}, \vartheta} \dot{\varphi}, \\
& \rho_{W}=\left.\frac{\partial \rho_{W}}{\partial P_{\vartheta}}\right|_{P_{\varphi}, \vartheta, \varphi} \dot{P}_{\vartheta}+\left.\frac{\partial \rho_{W}}{\partial P_{\varphi}}\right|_{P_{\vartheta}, \vartheta, \varphi} \dot{P}_{\varphi}+\left.\frac{\partial \rho_{W}}{\partial \vartheta}\right|_{P_{\vartheta}, P_{\varphi}, \varphi} \dot{\vartheta}+\left.\frac{\partial \rho_{W}}{\partial \varphi}\right|_{P_{\vartheta}, P_{\varphi}, \vartheta} \dot{\varphi} \\
& \dot{\mathcal{T}}=\left.\frac{\partial \mathcal{T}}{\partial t}\right|_{s, \vartheta, \varphi}+\left.\frac{\partial \mathcal{T}}{\partial s}\right|_{\vartheta, \varphi, t} \dot{s}+\left.\frac{\partial \mathcal{T}}{\partial \vartheta}\right|_{s, \varphi, t} \dot{\vartheta}+\left.\frac{\partial \mathcal{T}}{\partial \varphi}\right|_{s, \vartheta, t} \dot{\varphi}
\end{aligned}
$$

Consequently, writing the equations of motion in a form that is most easily comparable to the previously derived finite $\delta A_{\|}$only model, we obtain

$$
\begin{aligned}
& \dot{s}=\frac{\mu_{0} I(s)}{D}[(\left.\left.\frac{\mu}{e}+\sigma \tau \frac{e B}{m_{0}} \rho_{\|}^{2}\right) \frac{\partial B}{\partial \vartheta}+\left.\frac{\partial \Phi_{\mathrm{E}}}{\partial \vartheta}\right|_{s, \varphi, t}-\left.\frac{e \sigma^{2} B^{2}}{m_{0}} \rho_{\|} \frac{\partial \mathcal{T}}{\partial \vartheta}\right|_{s, \varphi, t}\right] \\
&+\frac{\mu_{0} J(s)}{D}\left[\left(\frac{\mu}{e}+\sigma \tau \frac{e B}{m_{0}} \rho_{\|}^{2}\right) \frac{\partial B}{\partial \varphi}+\left.\frac{\partial \Phi_{\mathrm{E}}}{\partial \varphi}\right|_{s, \vartheta, t}-\left.\frac{e \sigma^{2} B^{2}}{m_{0}} \rho_{\|} \frac{\partial \mathcal{T}}{\partial \varphi}\right|_{s, \vartheta, t}\right] \\
&-\left.\frac{e \sigma^{2} B^{2}}{m_{0} D} \rho_{\|} \frac{\partial \mathcal{W}}{\partial \varphi}\right|_{s, \vartheta, t}, \\
& \dot{\vartheta}=-\frac{\mu_{0} I(s)}{D}\left[\left(\frac{\mu}{e}+\sigma \tau \frac{e B}{m_{0}} \rho_{\|}^{2}\right) \frac{\partial B}{\partial s}+\left.\frac{e \sigma B^{2}}{m_{0}} \rho_{\|}^{2} \frac{\partial \sigma}{\partial s}\right|_{B}+\left.\frac{\partial \Phi_{\mathrm{E}}}{\partial s}\right|_{\vartheta, \varphi, t}-\left.\frac{e \sigma^{2} B^{2}}{m_{0}} \rho_{\|} \frac{\partial \mathcal{T}}{\partial s}\right|_{\vartheta, \varphi, t}\right] \\
&+\frac{e \sigma^{2} B^{2}}{m_{0} D} \rho_{\|}\left[\psi^{\prime}(s)+\mu_{0} I^{\prime}(s) \rho_{W}\right], \\
& \dot{\varphi}=-\frac{\mu_{0} J(s)}{D}\left[\left(\frac{\mu}{e}+\sigma \tau \frac{e B}{m_{0}} \rho_{\|}^{2}\right) \frac{\partial B}{\partial s}+\left.\frac{e \sigma B^{2}}{m_{0}} \rho_{\|}^{2} \frac{\partial \sigma}{\partial s}\right|_{B}+\left.\frac{\partial \Phi_{\mathrm{E}}}{\partial s}\right|_{\vartheta, \varphi, t}-\left.\frac{e \sigma^{2} B^{2}}{m_{0}} \rho_{\|} \frac{\partial \mathcal{T}}{\partial s}\right|_{\vartheta, \varphi, t}\right] \\
&+ \frac{e \sigma^{2} B^{2}}{m_{0} D} \rho_{\|}\left[\chi^{\prime}(s)+\mu_{0} J^{\prime}(s) \rho_{W}+\left.\frac{\partial \mathcal{W}}{\partial s}\right|_{\vartheta, \varphi, t}\right],
\end{aligned}
$$




$$
\begin{aligned}
\dot{\rho}_{\|}=-\left.\frac{\partial \mathcal{T}}{\partial t}\right|_{s, \vartheta, \varphi} & -\frac{1}{D}\left[\psi^{\prime}(s)+\mu_{0} I^{\prime}(s) \rho_{W}+\left.\mu_{0} I(s) \frac{\partial \mathcal{T}}{\partial s}\right|_{\vartheta, \varphi, t}\right] \\
\times & {\left[\left(\frac{\mu}{e}+\sigma \tau \frac{e B}{m_{0}} \rho_{\|}^{2}\right) \frac{\partial B}{\partial \vartheta}+\left.\frac{\partial \Phi_{\mathrm{E}}}{\partial \vartheta}\right|_{s, \varphi, t}\right] } \\
& -\frac{1}{D}\left[\chi^{\prime}(s)+\mu_{0} J^{\prime}(s) \rho_{W}+\left.\mu_{0} J(s) \frac{\partial \mathcal{T}}{\partial s}\right|_{\vartheta, \varphi, t}+\left.\frac{\partial \mathcal{W}}{\partial s}\right|_{\vartheta, \varphi, t}\right] \\
& \times\left[\left(\frac{\mu}{e}+\sigma \tau \frac{e B}{m_{0}} \rho_{\|}^{2}\right) \frac{\partial B}{\partial \varphi}+\left.\frac{\partial \Phi_{\mathrm{E}}}{\partial \varphi}\right|_{s, \vartheta, t}\right] \\
+ & \frac{1}{D}\left[\left.\mu_{0} I(s) \frac{\partial \mathcal{T}}{\partial \vartheta}\right|_{s, \varphi, t}+\left.\mu_{0} J(s) \frac{\partial \mathcal{T}}{\partial \varphi}\right|_{s, \vartheta, t}+\left.\frac{\partial \mathcal{W}}{\partial \varphi}\right|_{s, \vartheta, t}\right] \\
& \times\left[\left(\frac{\mu}{e}+\sigma \tau \frac{e B}{m_{0}} \rho_{\|}^{2}\right) \frac{\partial B}{\partial s}+\left.\frac{e \sigma B^{2}}{m_{0}} \rho_{\|}^{2} \frac{\partial \sigma}{\partial s}\right|_{B}+\left.\frac{\partial \Phi_{\mathrm{E}}}{\partial s}\right|_{\vartheta, \varphi, t}\right] .
\end{aligned}
$$

The finite $\delta A_{\|}$only model is directly recovered by imposing $\mathcal{T} \Rightarrow \Upsilon, \rho_{W} \Rightarrow \rho_{c}$ and $\mathcal{W} \Rightarrow 0$.

A more compact representation of the drift orbit equations is derived by substituting the expression for $\mathcal{T}$ and $\mathcal{W}$ in favour of $\delta A_{\vartheta}$ and $\delta A_{\varphi}$ which yields for the particle coordinate position

$$
\begin{aligned}
& \dot{s}=\frac{1}{D}\left[\left(\frac{\mu}{e}+\sigma \tau \frac{e B}{m_{0}} \rho_{\|}^{2}\right)\left[\mu_{0} I(s) \frac{\partial B}{\partial \vartheta}+\mu_{0} J(s) \frac{\partial B}{\partial \varphi}\right]\right. \\
& +\frac{1}{D}\left[\left.\mu_{0} I(s) \frac{\partial \Phi_{\mathrm{E}}}{\partial \vartheta}\right|_{s, \varphi, t}+\left.\mu_{0} J(s) \frac{\partial \Phi_{\mathrm{E}}}{\partial \varphi}\right|_{s, \vartheta, t}\right] \\
& +\frac{e \sigma^{2} B^{2}}{m_{0} D} \rho_{\|}\left[\left.\frac{\partial\left(\delta A_{\varphi}\right)}{\partial \vartheta}\right|_{s, \varphi, t}-\left.\frac{\partial\left(\delta A_{\vartheta}\right)}{\partial \varphi}\right|_{s, \vartheta, t}\right] \\
& \dot{\vartheta}=-\frac{\mu_{0} I(s)}{D}\left[\left(\frac{\mu}{e}+\sigma \tau \frac{e B}{m_{0}} \rho_{\|}^{2}\right) \frac{\partial B}{\partial s}+\left.\frac{e \sigma B^{2}}{m_{0}} \rho_{\|}^{2} \frac{\partial \sigma}{\partial s}\right|_{B}\right]+\frac{e \sigma^{2} B^{2}}{m_{0} D} \rho_{\|}\left[\psi^{\prime}(s)+\mu_{0} I^{\prime}(s) \rho_{\|}\right] \\
& -\left.\frac{\mu_{0} I(s)}{D} \frac{\partial \Phi_{\mathrm{E}}}{\partial s}\right|_{\vartheta, \varphi, t}-\left.\frac{e \sigma^{2} B^{2}}{m_{0} D} \rho_{\|} \frac{\partial\left(\delta A_{\varphi}\right)}{\partial s}\right|_{\vartheta, \varphi, t}, \\
& \dot{\varphi}=-\frac{\mu_{0} J(s)}{D}\left[\left(\frac{\mu}{e}+\sigma \tau \frac{e B}{m_{0}} \rho_{\|}^{2}\right) \frac{\partial B}{\partial s}+\left.\frac{e \sigma B^{2}}{m_{0}} \rho_{\|}^{2} \frac{\partial \sigma}{\partial s}\right|_{B}\right]+\frac{e \sigma^{2} B^{2}}{m_{0} D} \rho_{\|}\left[\chi^{\prime}(s)+\mu_{0} J^{\prime}(s) \rho_{\|}\right] \\
& -\left.\frac{\mu_{0} J(s)}{D} \frac{\partial \Phi_{\mathrm{E}}}{\partial s}\right|_{\vartheta, \varphi, t}+\left.\frac{e \sigma^{2} B^{2}}{m_{0} D} \rho_{\|} \frac{\partial\left(\delta A_{\vartheta}\right)}{\partial s}\right|_{\vartheta, \varphi, t}
\end{aligned}
$$

The convenience of this form lies in the fact that the motion in the equilibrium field, the electrostatic perturbed field and the electromagnetic perturbed field can be straightforwardly identified in each one of these equations. The evolution of the parallel gyroradius acquires the 
more complicated form

$$
\begin{aligned}
\dot{\rho}_{\|}=-\frac{1}{D}\left(\frac{\mu}{e}\right. & \left.+\sigma \tau \frac{e B}{m_{0}} \rho_{\|}^{2}\right)\left\{\left[\psi^{\prime}(s)+\mu_{0} I^{\prime}(s) \rho_{\|}\right] \frac{\partial B}{\partial \vartheta}+\left[\chi^{\prime}(s)+\mu_{0} J^{\prime}(s) \rho_{\|}\right] \frac{\partial B}{\partial \varphi}\right\} \\
& \left.-\frac{1}{D}\left\{\left.\left[\psi^{\prime}(s)+\mu_{0} I^{\prime}(s) \rho_{\|}\right] \frac{\partial \Phi_{\mathrm{E}}}{\partial \vartheta}\right|_{s, \varphi, t}+\left.\left[\chi^{\prime}(s)+\mu_{0} J^{\prime}(s) \rho_{\|}\right] \frac{\partial \Phi_{\mathrm{E}}}{\partial \varphi}\right|_{s, \vartheta, t}\right\} \mid\right] \\
& +\frac{1}{D}\left(\frac{\mu}{e}+\sigma \tau \frac{e B}{m_{0}} \rho_{\|}^{2}\right)\left[\left.\frac{\partial B}{\partial \vartheta} \frac{\partial\left(\delta A_{\varphi}\right)}{\partial s}\right|_{\vartheta, \varphi, t}-\left.\frac{\partial B}{\partial \varphi} \frac{\partial\left(\delta A_{\vartheta}\right)}{\partial s}\right|_{\vartheta, \varphi, t}\right] \\
& +\left.\frac{1}{\mu_{0} I(s)} \frac{\partial\left(\delta A_{\varphi}\right)}{\partial t}\right|_{s, \vartheta, \varphi} \mid \frac{1}{D}\left[\left(\frac{\mu}{e}+\sigma \tau \frac{e B}{m_{0}} \rho_{\|}^{2}\right) \frac{\partial B}{\partial s}+\left.\frac{e \sigma B^{2}}{m_{0}} \rho_{\|}^{2} \frac{\partial \sigma}{\partial s}\right|_{B}+\right]\left[\left.\frac{\partial\left(\delta A_{\varphi}\right)}{\partial \vartheta}\right|_{s, \varphi, t}-\left.\frac{\partial\left(\delta A_{\vartheta}\right)}{\partial \varphi}\right|_{s, \vartheta, t}\right] \\
& +\frac{1}{D}\left[\left.\left.\frac{\partial\left(\delta A_{\varphi}\right)}{\partial s}\right|_{\vartheta, \varphi, t} \frac{\partial \Phi_{\mathrm{E}}}{\partial \vartheta}\right|_{s, \varphi, t}-\left.\left.\frac{\partial\left(\delta A_{\vartheta}\right)}{\partial s}\right|_{\vartheta, \varphi, t} \frac{\partial \Phi_{\mathrm{E}}}{\partial \varphi}\right|_{s, \vartheta, t}\right] \\
& -\left.\frac{1}{D}\left[\left.\frac{\partial\left(\delta A_{\varphi}\right)}{\partial \vartheta}\right|_{s, \varphi, t}-\left.\frac{\partial\left(\delta A_{\vartheta}\right)}{\partial \varphi}\right|_{s, \vartheta, t}\right] \frac{\partial \Phi_{\mathrm{E}}}{\partial s}\right|_{\vartheta, \varphi, t} \cdot
\end{aligned}
$$

Again, the $\rho_{\|}$motion in the equilibrium, electrostatic and electromagnetic fields are easily identifiable. The last two terms constitute the nonlinear drive due essentially to wave-wave interactions specifically involving the electrostatic field acting on the electromagnetic field oscillations.

\section{The weights in the $\delta f$ formulation}

The guiding centre drift orbit equations derived constitute an essential feature of a Monte-Carlo $\delta f$ solution of the drift kinetic equation using a numerical particle method. Formally, the drift kinetic equation can be written as

$$
\frac{\mathrm{d} f}{\mathrm{~d} t}=0
$$

where the full time derivative is

$$
\frac{\mathrm{d}}{\mathrm{d} t}=\frac{\partial}{\partial t}+\dot{s} \frac{\partial}{\partial s}+\dot{\vartheta} \frac{\partial}{\partial \vartheta}+\dot{\varphi} \frac{\partial}{\partial \varphi}+\dot{\rho_{\|}} \frac{\partial}{\partial \rho_{\|}}+\dot{\epsilon} \frac{\partial}{\partial \epsilon}+\dot{\mu} \frac{\partial}{\partial \mu} .
$$

Invoking a $\delta f$ formalism, we write $f=F_{0}+\delta f$ in which $F_{0}$ and $\delta f$ are the stationary background and fluctuating components (assuming $\delta f \ll F_{0}$ ) of the full particle distribution function from which we obtain using equation (46) [13]

$$
\frac{\mathrm{d} \delta f}{\mathrm{~d} t}=-\frac{\mathrm{d} F_{0}}{\mathrm{~d} t},
$$

and we approximate $\delta f \simeq \sum_{N} w_{N}(t) \delta\left(z-z_{N}(t)\right)$, where $N$ is the marker (or numerical) particle label, $w_{N}$ represents the marker weight of each particle, $z=\left(s, \vartheta, \varphi, \rho_{\|}, \epsilon, \mu\right)$ and $\mathrm{d} z_{N} / \mathrm{d} t$ corresponds to the physical trajectories of the particles. The evolution of the weights $w_{N}$ then reads as $[21,22]$

$$
\frac{\mathrm{d} w_{N}}{\mathrm{~d} t}=-\left.\alpha_{N} \frac{\mathrm{d} F_{0}}{\mathrm{~d} t}\right|_{\left(Z_{N}, t\right)}
$$


where $\alpha_{N}$ stands for the constant phase space volume associated with each marker. The background distribution function $F_{0}$ is most conveniently, but not necessarily, expressed as a function of the constants of the motion so as to represent an equilibrium distribution. Thus, in axisymmetric or nearly axisymmetric systems $F_{0}=F_{0}\left(P_{\varphi}, \epsilon, \mu\right)$ the evolution of the weights $w$ of each marker particle can be expressed as [13,21,22]

$$
\frac{\mathrm{d} w}{\mathrm{~d} t}=-\alpha\left[\left.\left.\frac{\mathrm{d} P_{\varphi}}{\mathrm{d} t}\right|_{p} \frac{\partial F_{0}}{\partial P_{\varphi}}\right|_{\epsilon, \mu}+\left.\left.\frac{\mathrm{d} \epsilon}{\mathrm{d} t}\right|_{p} \frac{\partial F_{0}}{\partial \epsilon}\right|_{P_{\varphi}, \mu}\right],
$$

where the subscript $p$ indicates that the variation in the perturbed fields needs only to be considered. For simplicity, we have dropped the subscript $N$ as the label for all marker quantities. The equilibrium distribution function of a particular species of interest in anisotropic pressure equilibria may also be a function of the magnetic moment $\mu$. In the drift approximation, as illustrated in equation (10), the wavelengths of the perturbed fields must be comparable to or exceed the dimensions of the ion Larmor radii and must evolve slower than the inverse particle gyrofrequency. Under these conditions $\mathrm{d} \mu / \mathrm{d} t=0$ is satisfied. The contours of the second adiabatic invariant $\mathcal{J} \equiv \oint v_{\|} \mathrm{d} \ell$ close poloidally for all trapped particles in quasi-isodynamic stellarators [23]. Therefore $\mathcal{J}$ constitutes a variable that is very close to a constant of the motion in such systems, so $F_{0}=F_{0}(\mathcal{J}, \epsilon, \mu)$ is chosen and the evolution of $w$ is given by

$$
\frac{\mathrm{d} w}{\mathrm{~d} t}=-\alpha\left[\left.\left.\frac{\mathrm{d} \mathcal{J}}{\mathrm{d} t}\right|_{p} \frac{\partial F_{0}}{\partial \mathcal{J}}\right|_{\epsilon, \mu}+\left.\left.\frac{\mathrm{d} \epsilon}{\mathrm{d} t}\right|_{p} \frac{\partial F_{0}}{\partial \epsilon}\right|_{\mathcal{J}, \mu}\right] .
$$

However, for conventional stellarators, the poloidal closure of the $\mathcal{J}$ contours occurs only for a very limited fraction of the trapped particle population, so the expression given in equation (51) may not be the most relevant to apply. In this case one may simply consider $F_{0}=F_{0}(s, \epsilon, \mu)$ (note that in this case $F_{0}$ is not necessarily an equilibrium distribution as the radial variable $s$ is not a constant of the motion). For such a class of stellarators, the evolution equation for the weights becomes

$$
\frac{\mathrm{d} w}{\mathrm{~d} t}=-\alpha\left[\left.\frac{\mathrm{d} s}{\mathrm{~d} t} \frac{\partial F_{0}}{\partial s}\right|_{\epsilon, \mu}+\left.\left.\frac{\mathrm{d} \epsilon}{\mathrm{d} t}\right|_{p} \frac{\partial F_{0}}{\partial \epsilon}\right|_{s, \mu}\right] .
$$

Note that the full time derivative of the radial variable $s$ needs to be considered in this case. When, as in a 3D equilibrium system, the exact constants of the motion are not known precisely, $\delta f$ represents not only the fluctuating component of the full- $f$, but also the complement of the $F_{0}$ specified with respect to an exact equilibrium distribution. Given a sufficiently large marker population, the numerical system converges towards an exact solution. To preserve the advantages of the $\delta f$ over the full- $f$ method, the condition $\delta f \ll F_{0}$ must be ensured.

To determine the time evolution of the particle energy $\epsilon=\mathcal{H}-e \Phi_{\mathrm{E}}$, we must evaluate first the evolution of the Hamiltonian which is given by

$$
\frac{\mathrm{d} \mathcal{H}}{\mathrm{d} t}=\left.e \frac{\partial \Phi_{\mathrm{E}}}{\partial t}\right|_{s, \vartheta, \varphi}-\left.\frac{e^{2} \sigma^{2} B^{2}}{m_{0}} \rho_{\|} \frac{\partial \mathcal{T}}{\partial t}\right|_{s, \vartheta, \varphi} .
$$

Using equation (30) we get

$$
\begin{aligned}
\left.\frac{\mathrm{d} \epsilon}{\mathrm{d} t}\right|_{p}=\frac{\mathrm{d} \mathcal{H}}{\mathrm{d} t}- & e \frac{\mathrm{d} \Phi_{\mathrm{E}}}{\mathrm{d} t}=-\left.\frac{e^{2} \sigma^{2} B^{2}}{m_{0}} \rho_{\|} \frac{\partial \mathcal{T}}{\partial t}\right|_{s, \vartheta, \varphi} \\
& -\left.e \frac{\partial \Phi_{\mathrm{E}}}{\partial s}\right|_{\vartheta, \varphi, t} \frac{\mathrm{d} s}{\mathrm{~d} t}-\left.e \frac{\partial \Phi_{\mathrm{E}}}{\partial \vartheta}\right|_{s, \varphi, t} \frac{\mathrm{d} \vartheta}{\mathrm{d} t}-\left.e \frac{\partial \Phi_{\mathrm{E}}}{\partial \varphi}\right|_{s, \vartheta, t} \frac{\mathrm{d} \varphi}{\mathrm{d} t} .
\end{aligned}
$$


Note that the particle energy is conserved in the absence of perturbed fields. As the toroidal angular momentum is given by $P_{\varphi}=\psi+\mu_{0} I(s)\left(\rho_{\|}+\mathcal{T}\right)$, we can write its time evolution as

$$
\begin{aligned}
\frac{\mathrm{d} P_{\varphi}}{\mathrm{d} t}=\left[\psi^{\prime}(s)\right. & \left.+\mu_{0} I^{\prime}(s)\left(\rho_{\|}+\mathcal{T}\right)+\left.\mu_{0} I(s) \frac{\partial \mathcal{T}}{\partial s}\right|_{\vartheta, \varphi, t}\right] \frac{\mathrm{d} s}{\mathrm{~d} t}+\mu_{0} I(s) \frac{\mathrm{d} \rho_{\|}}{\mathrm{d} t} \\
& +\left.\mu_{0} I(s) \frac{\partial \mathcal{T}}{\partial \vartheta}\right|_{s, \varphi, t} \frac{\mathrm{d} \vartheta}{\mathrm{d} t}+\left.\mu_{0} I(s) \frac{\partial \mathcal{T}}{\partial \varphi}\right|_{s, \vartheta, t} \frac{\mathrm{d} \varphi}{\mathrm{d} t}+\left.\mu_{0} I(s) \frac{\partial \mathcal{T}}{\partial t}\right|_{s, \vartheta, \varphi} .
\end{aligned}
$$

Discarding the motion in the equilibrium fields, we can then write

$$
\begin{array}{r}
\left.\frac{\mathrm{d} P_{\varphi}}{\mathrm{d} t}\right|_{p}=\left.\left[\psi^{\prime}(s)+\mu_{0} I^{\prime}(s) \rho_{\|}\right] \frac{\mathrm{d} s}{\mathrm{~d} t}\right|_{p}+\left.\mu_{0} I(s) \frac{\mathrm{d} \rho_{\|}}{\mathrm{d} t}\right|_{p}-\left.\frac{\partial\left(\delta A_{\varphi}\right)}{\partial s}\right|_{\vartheta, \varphi, t} \frac{\mathrm{d} s}{\mathrm{~d} t} \\
-\left.\frac{\partial\left(\delta A_{\varphi}\right)}{\partial \vartheta}\right|_{s, \varphi, t} \frac{\mathrm{d} \vartheta}{\mathrm{d} t}-\left.\frac{\partial\left(\delta A_{\varphi}\right)}{\partial \varphi}\right|_{s, \vartheta, t} \frac{\mathrm{d} \varphi}{\mathrm{d} t}-\left.\frac{\partial\left(\delta A_{\varphi}\right)}{\partial t}\right|_{s, \vartheta, \varphi} .
\end{array}
$$

\section{Implications of Faraday's law for MHD structures}

Faraday's Law is

$$
\boldsymbol{E}=-\frac{\partial(\delta \boldsymbol{A})}{\partial t}-\nabla \Phi_{\mathrm{E}}
$$

In the MHD representation, $\delta \boldsymbol{A}_{\mathrm{MHD}}=\boldsymbol{\xi} \times \boldsymbol{B}$ and $\Phi_{\mathrm{EMHD}}=0$. The vector $\boldsymbol{\xi}$ corresponds to the perturbed MHD displacement vector and is typically expressed as

$$
\xi=\sqrt{g} \xi^{s} \nabla \vartheta \times \nabla \varphi+\frac{B \times \nabla s}{B^{2}} \eta .
$$

The condition $\boldsymbol{E} \cdot \boldsymbol{B}=0$ is thus trivially satisfied for MHD perturbations. Regardless of a change of gauge condition, $\boldsymbol{E} \cdot \boldsymbol{B}=0$ when MHD electromagnetic fields are investigated. Thus in the drift representation we have

$$
\boldsymbol{E} \cdot \boldsymbol{B}=-\frac{\partial\left(\boldsymbol{B} \cdot \delta \boldsymbol{A}_{D}\right)}{\partial t}-\boldsymbol{B} \cdot \nabla \Phi_{\mathrm{E}}=0 .
$$

Essentially, this yields a relation between the electrostatic potential and the parallel component of the electromagnetic potential when MHD modes are considered.

\section{Potential structure for the finite $\delta A_{\|}$only model}

The determination of the potentials when only finite $\delta A_{\|}$is retained has been discussed extensively in previous work $[2,13,21]$, but for the sake of completeness we review it here. Therefore, under such conditions $\delta \boldsymbol{A}=\Upsilon \sigma \boldsymbol{B}$ and from Faraday's law we get

$$
\left.\sigma B^{2} \frac{\partial \Upsilon}{\partial t}\right|_{s, \vartheta, \varphi}+B \cdot \nabla \Phi_{\mathrm{E}}=0
$$

In order to make further progress, the equivalence of the perturbed radial magnetic field is usually invoked in order to guarantee that the radial drift motion is accurately described [2]. This is not unique, as the application of the equivalence of other projections of the perturbed magnetic field could have been examined at the cost of a more inaccurate determination of the radial motion of the drift orbit. Thus, in the MHD representation, we have

$$
\delta \boldsymbol{B} \cdot \nabla s=\nabla s \cdot \nabla \times(\boldsymbol{\xi} \times \boldsymbol{B})=(\boldsymbol{B} \cdot \nabla) \xi^{s} .
$$


In the drift representation, on the other hand, we get

$$
\delta \boldsymbol{B} \cdot \nabla s=\nabla s \cdot \nabla \times(\Upsilon \sigma \boldsymbol{B})=\sigma \boldsymbol{B} \times \nabla s \cdot \nabla \Upsilon
$$

which then yields the relation

$$
\sigma \boldsymbol{B} \times \nabla s \cdot \nabla \Upsilon=(\boldsymbol{B} \cdot \nabla) \xi^{s} .
$$

\section{Fourier space solution for the finite $\delta A_{\|}$only model}

We expand the perturbed field components in a Fourier series as follows:

$$
\begin{aligned}
& \xi^{s}(s, \vartheta, \varphi, t)=\sum_{m n} \xi_{m n}^{s}(s) \sin (m \vartheta-n \varphi+\Delta-\omega t), \\
& \Upsilon(s, \vartheta, \varphi, t)=\sum_{m n} \Upsilon_{m n}(s) \sin (m \vartheta-n \varphi+\Delta-\omega t), \\
& \Phi_{\mathrm{E}}(s, \vartheta, \varphi, t)=\sum_{m n} \Phi_{\mathrm{E} m n}(s) \sin (m \vartheta-n \varphi+\Delta-\omega t),
\end{aligned}
$$

where $\omega$ is the mode frequency (typically of an Alfvén mode), $m$ is the poloidal mode number, $n$ is the toroidal mode number and $\Delta$ is a phase factor. The Fourier solutions for the wave fields from Faraday's law yields

$$
\Phi_{\mathrm{E} m n}(s)=\omega\left[\frac{\sigma \sqrt{g} B^{2}}{m \psi^{\prime}(s)-n \chi^{\prime}(s)}\right] \Upsilon_{m n}(s),
$$

and from the perturbed radial magnetic field projection we get

$$
\Upsilon_{m n}(s)=-\frac{1}{\mu_{0}}\left[\frac{m \psi^{\prime}(s)-n \chi^{\prime}(s)}{m I(s)-n J(s)}\right] \xi_{m n}^{s}(s),
$$

from which we derive the relation

$$
\Phi_{\mathrm{E} m n}(s)=-\frac{\omega}{\mu_{0}}\left[\frac{\sigma \sqrt{g} B^{2}}{m I(s)-n J(s)}\right] \xi_{m n}^{s}(s) .
$$

\section{Potential structure for full electromagnetic fields}

The examination of drift orbits in the presence of full electromagnetic fields does not require the evaluation of the radial component of the perturbed magnetic field to assure relationships between projections of the vector potential and the electrostatic potential as in the reduced $\delta A_{\|}$only model. One key feature is the recognition that the vector potential is only known up to the gradient of a scalar function to reproduce any perturbed magnetic field. Hence we can write

$$
\delta \boldsymbol{A}_{\mathrm{MHD}}=\delta \boldsymbol{A}_{\mathrm{D}}+\nabla G
$$

from which we extract the parallel component as

$$
\boldsymbol{B} \cdot \delta \boldsymbol{A}_{\mathrm{MHD}}=\boldsymbol{B} \cdot \delta \boldsymbol{A}_{\mathrm{D}}+\boldsymbol{B} \cdot \nabla G=0 .
$$

Taking the derivative with respect to $t$ and equating terms with the parallel projection of Faraday's law yields

$$
\Phi_{\mathrm{E}}=\frac{\partial G}{\partial t}
$$


The radial projection $\sqrt{g} \nabla \vartheta \times \nabla \varphi \cdot \delta A$, invoking the gauge condition $\delta A_{s}=0$, gives

$$
\left.\frac{\partial \Phi_{\mathrm{E}}}{\partial s}\right|_{\vartheta, \varphi, t}=\left.\frac{\partial \eta}{\partial t}\right|_{s, \vartheta, \varphi},
$$

the poloidal projection $\sqrt{g} \nabla \varphi \times \nabla s \cdot \delta A$ reads as

$$
\left.\frac{\partial\left(\delta A_{\vartheta}\right)}{\partial t}\right|_{s, \vartheta, \varphi}=-\left.\frac{\partial \Phi_{\mathrm{E}}}{\partial \vartheta}\right|_{s, \varphi, t}-\left.\chi^{\prime}(s) \frac{\partial \xi^{s}}{\partial t}\right|_{s, \vartheta, \varphi},
$$

while the toroidal projection $\sqrt{g} \nabla s \times \nabla \vartheta \cdot \delta \boldsymbol{A}$ produces

$$
\left.\frac{\partial\left(\delta A_{\varphi}\right)}{\partial t}\right|_{s, \vartheta, \varphi}=-\left.\frac{\partial \Phi_{\mathrm{E}}}{\partial \varphi}\right|_{s, \vartheta, t}+\left.\psi^{\prime}(s) \frac{\partial \xi^{s}}{\partial t}\right|_{s, \vartheta, \varphi} .
$$

One important difference between the full electromagnetic model and the reduced finite $\delta A_{\|}$ only model is that $\Phi_{\mathrm{E}}$ is related to the binormal component of the MHD displacement vector $\eta$ instead of its radial component $\xi^{s}$. The procedure is to solve first for $\Phi_{\mathrm{E}}$ in terms of $\eta$. Once this solution is obtained, we proceed with expressions for the electromagnetic vector potential components $\delta A_{\vartheta}$ and $\delta A_{\varphi}$.

\section{Fourier space solution for full electromagnetic fields}

The Fourier expansion of the field components $\xi^{s}$ and $\Phi_{\mathrm{E}}$ has already been presented in section 9. The remaining field components are given by

$$
\begin{aligned}
& \eta(s, \vartheta, \varphi, t)=\sum_{m n} \eta_{m n}(s) \cos (m \vartheta-n \varphi+\Delta-\omega t), \\
& \delta A_{\varphi}(s, \vartheta, \varphi, t)=\sum_{m n} \delta A_{\varphi m n}(s) \sin (m \vartheta-n \varphi+\Delta-\omega t), \\
& \delta A_{\vartheta}(s, \vartheta, \varphi, t)=\sum_{m n} \delta A_{\vartheta m n}(s) \sin (m \vartheta-n \varphi+\Delta-\omega t) .
\end{aligned}
$$

Consequently, the Fourier solutions for the wave field amplitudes are

$$
\begin{aligned}
& \frac{\mathrm{d} \Phi_{\mathrm{E} m n}}{\mathrm{~d} s}=\omega \eta_{m n}(s), \\
& \delta A_{\varphi m n}(s)=\psi^{\prime}(s) \xi_{m n}^{s}(s)-\frac{n}{\omega} \Phi_{\mathrm{E} m n}(s), \\
& \delta A_{\vartheta m n}(s)=-\chi^{\prime}(s) \xi_{m n}^{s}(s)+\frac{m}{\omega} \Phi_{\mathrm{E} m n}(s) .
\end{aligned}
$$

As described earlier, we first perform the radial integral to determine $\Phi_{\mathrm{E} m n}(s)$ from $\eta_{m n}(s)$ and then proceed to algebraically evaluate $\delta A_{\varphi m n}(s)$ and $\delta A_{\vartheta m n}(s)$. The radial derivatives of $\delta A_{\varphi m n}(s)$ and $\delta A_{\vartheta m n}(s)$ with respect to $s$ should be computed directly from the MHD fields, namely

$$
\begin{aligned}
& \frac{\mathrm{d}\left(\delta A_{\varphi m n}\right)}{\mathrm{d} s}=\psi^{\prime \prime}(s) \xi_{m n}^{s}(s)+\psi^{\prime}(s) \frac{\mathrm{d} \xi_{m n}^{s}}{\mathrm{~d} s}-n \eta_{m n}(s), \\
& \frac{\mathrm{d}\left(\delta A_{\vartheta m n}\right)}{\mathrm{d} s}=-\chi^{\prime \prime}(s) \xi_{m n}^{s}(s)-\chi^{\prime}(s) \frac{\mathrm{d} \xi_{m n}^{s}}{\mathrm{~d} s}+m \eta_{m n}(s) .
\end{aligned}
$$




\section{Summary and conclusions}

A Hamiltonian/Lagrangian theory is extended to describe guiding centre particle drift motion to include full electrostatic and electromagnetic field perturbations in 3D magnetic confinement geometry with nested flux surfaces. The formulation allows for anisotropic pressure caused by energetic particles driven by auxiliary heating systems. The Lagrangian formalism is a very useful tool to help identify the canonical momenta and angular variables. A partial redefinition of the guiding centre velocity is invoked to eliminate the radial motion associated with finite equilibrium radial magnetic field effects and is an important step in the definition of the canonical variables. The imposition of a gauge condition that eliminates the radial component of the perturbed electromagnetic potential in the covariant representation constitutes a crucial step that guarantees the Boozer magnetic coordinate angles correspond to a canonical frame for the drift motion. Following the evolution of the canonical momenta is less intuitive than the guiding centre particle radial motion and its parallel gyroradius. Thus a transformation is derived in a form that permits a straightforward comparison with a previously derived model that retains only finite $\delta A_{\|}$components. A more concise form of the drift orbits in which the poloidal and toroidal components of $\delta \boldsymbol{A}$ in the covariant representation explicitly appear demonstrates that the guiding centre particle coordinate positions are affected by wave-particle interactions in a linear fashion. The nonlinear wave-wave interactions (specifically involving the interaction of the electrostatic potential with components of the electromagnetic potential) only explicitly alter the evolution of the parallel gyroradius. The evolution of the particle weights for a Monte-Carlo $\delta f$ formalism like that applied in the VENUS code [24] is addressed.

The investigation of MHD-like mode structures on drift orbit motion requires the application of Faraday's law to connect the electrostatic potential with the parallel component of the electromagnetic potential. With full electromagnetic fields, equating the perturbed radial magnetic field with different representations of the vector potential is no longer required (as in the case in which only finite $\delta A_{\|}$is retained). Then, taking into consideration that the vector potential is only known to within the gradient of a scalar function, we can use Faraday's law to relate the electrostatic potential to the time variation of this scalar function. The radial projection of the vector potential in the MHD and drift representations, invoking the gauge condition, equates the radial derivative of the electrostatic potential to the time variation of the binormal component of the MHD displacement vector. Note that in the finite $\delta A_{\|}$only model, the electrostatic potential is expressed in terms of the radial component of the displacement vector. Once the electrostatic potential has been determined, we subsequently can obtain expressions for the two relevant components of the electromagnetic potential in terms of $\Phi_{\mathrm{E}}$ and the radial component of the MHD displacement vector. These manipulations are shown to be relatively simple with a Fourier decomposition of the perturbed variables.

\section{Acknowledgments}

This research was partially sponsored by the Fonds National Suisse de la Recherche Scientifique and Euratom.

\section{Appendix}

In order to evaluate the derivatives of the Hamiltonian with respect to the canonical variables, we must determine the corresponding derivatives of $s$ and $\rho_{W}$. This is realized by taking 
different cross derivatives of the canonical momenta and angles with respect to one another to obtain

$$
\begin{aligned}
\left.\frac{\partial s}{\partial P_{\vartheta}}\right|_{P_{\varphi}, \vartheta, \varphi} & =-\frac{\mu_{0} I(s)}{D},\left.\quad \frac{\partial \rho_{W}}{\partial P_{\vartheta}}\right|_{P_{\varphi}, \vartheta, \varphi}=\frac{\psi^{\prime}(s)+\rho_{W} \mu_{0} I^{\prime}(s)}{D}, \\
\left.\frac{\partial s}{\partial P_{\varphi}}\right|_{P_{\vartheta}, \vartheta, \varphi} & =-\frac{\mu_{0} J(s)}{D},\left.\quad \frac{\partial \rho_{W}}{\partial P_{\varphi}}\right|_{P_{\vartheta}, \vartheta, \varphi}=\frac{1}{D}\left[\chi^{\prime}(s)+\rho_{W} \mu_{0} J^{\prime}(s)+\left.\frac{\partial \mathcal{W}}{\partial s}\right|_{\vartheta, \varphi, t}\right], \\
\left.\frac{\partial s}{\partial \vartheta}\right|_{P_{\vartheta}, P_{\varphi}, \varphi} & =\left.\left[\frac{\mu_{0} I(s)}{D}\right] \frac{\partial \mathcal{W}}{\partial \vartheta}\right|_{s, \varphi, t},\left.\quad \frac{\partial \rho_{W}}{\partial \vartheta}\right|_{P_{\vartheta}, P_{\varphi}, \varphi}=\left.\left[\frac{\psi^{\prime}(s)+\rho_{W} \mu_{0} I^{\prime}(s)}{D}\right] \frac{\partial \mathcal{W}}{\partial \vartheta}\right|_{s, \varphi, t}, \\
\left.\frac{\partial s}{\partial \varphi}\right|_{P_{\vartheta}, P_{\varphi}, \vartheta} & =\left.\left[\frac{\mu_{0} I(s)}{D}\right] \frac{\partial \mathcal{W}}{\partial \varphi}\right|_{s, \vartheta, t},
\end{aligned}
$$

The denominator $D$ is given by

$$
D \equiv \mu_{0}\left[\psi^{\prime}(s) J(s)-\chi^{\prime}(s) I(s)\right]\left[1+\mu_{0} \rho_{W} \frac{J(s) I^{\prime}(s)-I(s) J^{\prime}(s)}{\psi^{\prime}(s) J(s)-\chi^{\prime}(s) I(s)}\right]-\left.\mu_{0} I(s) \frac{\partial \mathcal{W}}{\partial s}\right|_{\vartheta, \varphi, t} .
$$

(C) Euratom 2011.

\section{References}

[1] Boozer A H 1980 Phys. Fluids 23904

[2] White R B and Chance M S 1984 Phys. Fluids 272455

[3] Yamaguchi T et al 2005 Nucl. Fusion 45 L33

[4] Madden N A and Hastie R J 1994 Nucl. Fusion 34519

[5] Zwingmann W, Eriksson L G and Stubberfield P 2001 Plasma Phys. Control. Fusion 431441

[6] Cooper W A et al 2005 Plasma Phys. Control. Fusion 47561

[7] Cooper W A et al 2006 Nucl. Fusion 46683

[8] White R B, Boozer A H and Hay R 1982 Phys. Fluids 25575

[9] Cooper W A 1997 Plasma Phys. Control. Fusion 39931

[10] Cooper W A, Graves J P, Jucker M and Isaev M Yu 2006 Phys. Plasmas 13092501

[11] Littlejohn R G 1985 Phys. Fluids 282015

[12] White R B 1990 Phys. Fluids B 2845

[13] Pinches S D et al 1998 Comput. Phys. Commun. 111133

[14] Wang S 2006 Phys. Plasmas 13052506

[15] Cooper G A, Jucker M, Cooper W A, Graves J P and Isaev M Yu 2007 Phys. Plasmas 14102506

[16] Meiss J D and Hazeltine R D 1990 Phys. Fluids B 22563

[17] White R and Zakharov L E 2003 Phys. Plasmas 10573

[18] Grad H 1966 Phys. Fluids 9498

[19] Pustovitov V D 2010 Plasma Phys. Control. Fusion 52065001

[20] Cooper W A et al 2009 Comput. Phys. Commun. 1801524

[21] Chen Y, White R B, Fu G Y and Nazikian R 1999 Phys. Plasmas 6226

[22] Todo Y 2006 Phys. Plasmas 13082503

[23] Mikhailov M I, Shafranov V D, Subbotin A A, Isaev M Yu, Nührenberg J, Zille R and Cooper W A 2002 Nucl. Fusion $\mathbf{4 2}$ L23

[24] Fischer O, Cooper W A, Isaev M Yu and Villard L 2000 Nucl. Fusion 2817 